Pathologe 2011 · 32:273-274

DOI 10.1007/s00292-011-1431-5

Online publiziert: 12. Juni 2011

(c) Springer-Verlag 2011

\author{
G. Baretton \\ Institut für Pathologie, Universitätsklinikum „Carl Gustav Carus“, TU Dresden
}

\title{
Pathologie des unteren Gastrointestinaltrakts
}

Einsendungen aus dem Gastrointestinaltrakt machen einen Großteil des Untersuchungsguts in den meisten Instituten für Pathologie aus. Im vorliegenden Schwerpunktheft „Unterer Gastrointestinaltrakt“ soll praxisorientiert ein Überblick über aktuelle Entwicklungen und Standards bei der Befundung von Biopsien und Resektaten aus dem Kolorektum und seinen Grenzregionen gegeben werden. Dabei wird auf die aktualisierte WHO-Klassifikation der gastrointestinalen Tumoren (4. Aufl., November 2010) und die 7. Auflage der UICC-/TNM-Klassifikation (gültig ab 2010) Bezug genommen. Besonders wurde darauf geachtet, auch „Problemzonen“ in der Diagnostik darzustellen und zu erläutern, die in der täglichen Routine immer wieder Schwierigkeiten bereiten.

So befasst sich der Beitrag „Mikroskopische Kolitis“"von C. Platz-Baudin et al. mit den histologischen Diagnosekriterien und den klinisch-pathologischen Korrelationen. Neben dem klinischen Leitsymptom einer wässrigen Diarrhö zeigt sich hier eine charakteristische Histologie. Da die mikroskopische Kolitis definitionsgemäß einen endoskopischen Normalbefund zeigt, bleibt die Diagnosesicherung somit der Histomorphologie vorbehalten. Die beiden morphologischen Formen der mikroskopischen Kolitis, die lymphozytäre und die kollagene Kolitis, werden dargestellt und die entsprechenden Diagnosekriterien erläutert.

Das sehr problematische Gebiet der histomorphologischen Differenzialdiagnostik von „polypoiden Dysplasien bei chronisch-entzündlicher Darmerkrankung" wird in der Übersicht von $S$. Warich-Eitel et al. beschrieben. Dabei werden z. B. auch neue Erkenntnisse über „Adenoma-like-DALM“ aufgezeigt, bei denen trotz der Entstehung der dysplastischen Veränderungen auf dem Boden einer chronisch-entzündlichen Darmerkrankung überlappende morphologische Befunde mit sporadischen Adenomen vorliegen. Für die Patienten bedeutet dies, dass bei diesen Läsionen im Gegensatz zur klassischen DALM keine Proktokolektomie erfolgen muss, sondern offenbar eine lokale Abtragung als therapeutische Konsequenz ausreicht.

Für das klinische Management von $\mathrm{Pa}$ tienten mit endoskopisch abgetragenen kolorektalen Polypen erwartet der Kliniker bzw. Gastroenterologe einen histopathologischen Befund, aus dem sich alle relevanten Informationen schnell und einfach entnehmen lassen. Die Arbeit von G. Baretton et al. präsentiert eine Checkliste für „die standardisierte und strukturierte histologische Befundung kolorektaler Polypen vor dem Hintergrund der neuen WHO-Klassifikation“, die kürzlich von einer Taskforce aus Gastroenterologen und Pathologen erarbeitet wurde und helfen soll, sowohl die endoskopische als auch die pathohistologische Befundung zu verbessern und zu vereinheitlichen.

Nichtneoplastische und nichthamartomatöse Polypen im Kolorektum bereiten immer wieder diagnostische Probleme. In dem Artikel vom D.E. Aust u. J. Rüschoff werden sowohl die wichtigsten Vertreter dieser Gruppe, d. h. Polypen bei Mukosaprolapssyndrom und inflammatorische Polypen, als auch seltenere tumorähnliche Läsionen, wie Schleimhautheterotopien, Endometriosis externa etc., dargestellt und die wichtigsten Differenzialdiagnosen diskutiert.

Außer der Klassifikation kolorektaler Polypen sollte der Pathologe stets an die
Möglichkeit einer evtl. zugrunde liegenden Polypose denken. In dem Beitrag von A. Roessner et al. zum Thema „Kolorektale Polyposen - eine Anleitung zur Diagnostik" werden phänotypische Merkmale und genetische Grundlagen der wichtigsten Polyposesyndrome erläutert. Da sich aus der Morphologie einzelner Läsionen sowie deren Anzahl und Verteilung unter Umständen Rückschlüsse auf einen hereditären Hintergrund ziehen lassen, kommt der histomorphologischen Diagnose im Rahmen einer genetischen Beratung des Patienten und seiner Familienangehörigen große Bedeutung zu.

Die adäquate Diagnose und Therapie der neuroendokrinen Neoplasien der Appendix und des Kolorektums setzen eine genaue Kenntnis ihrer Klassifikation nach WHO und TNM voraus. In den Neuauflagen beider Klassifikationen haben sich auf diesem Gebiet wichtige Änderungen bzw. Neuerungen ergeben, die in dem Beitrag von G. Klöppel u. H. Scherübl prägnant dargestellt werden.

Eine verbesserte chirurgische Technik, speziell die Einführung der totalen mesorektalen Exzision (TME), hat zu einer signifikanten Steigerung der Heilungsraten und $\mathrm{zu}$ einer deutlichen Reduktion der pelvinen Lokalrezidive geführt. Dem Pathologen kommt bei der Beurteilung der Qualität der Operationspräparate dabei eine Schlüsselrolle zu. K.J. Schmitz et al. gehen in ihrem Artikel „Pathologische Aufarbeitung von Rektumkarzinomen mit partieller/totaler mesorektaler Exzision" speziell auf die Problematik von Resektionsgrenzen, Lymphknotenpräparation und Effekten einer neoadjuvanten Therapie ein.

Auch bei der Therapie kolorektaler Lebermetastasen haben sich die therapeuti- 
schen Strategien in den letzten Jahren grundlegend verändert. Die präadjuvante Chemotherapie stellt mittlerweile eine nicht mehr wegzudenkende Therapieoption für Patienten dar, die als Folge eines kolorektalen Karzinoms Lebermetastasen entwickelt haben. Durch die präoperative Regression der Metastasen kann sich die Prognose entscheidend verbessern, jedoch können die Chemotherapeutika auch Kollateralschäden am Leberparenchym verursachen, insbesondere wenn eine Vorschädigung des Lebergewebes vorliegt. Die Risiken von Chemotherapeutika für das Leberparenchym, wie das sinusoidale Obstruktionssyndrom, die noduläre regenerative Hyperplasie und die weniger bekannte chemotherapieassoziierte Steatohepatitis werden in dem Beitrag „Steatohepatitis bei der Chemotherapie kolorektaler Lebermestastasen (CASH)" von A. Tannapfel et al. ausführlich erläutert.

Schließlich liefern J. Rüschoff et al. mit ihrer Arbeit „Das Analkarzinom - diagnostische und differenzialdiagnostische Aspekte" einen Überblick über das breite Differenzierungsspektrum primärer Analkarzinome, das von drüsiger Rektumschleimhaut über urothelartiges Epithel an der Linea dentata zu unverhorntem analem Plattenepithel und schließlich in die perianale Haut sowie speziellen proktodäalen/analen Drüsen reicht.

Herausgeber und Autoren hoffen, mit diesem Schwerpunktheft praktische Hilfen in der pathomorphologischen Diagnostik und Differenzialdiagnostik von Erkrankungen des unteren Gastrointestinaltrakts zu geben und darüber hinaus einen Beitrag zur Standardisierung der histopathologischen Befunde in dieser Region zu leisten.

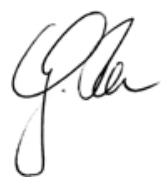

G. Baretton

\section{Korrespondenzadresse}

\section{Prof. Dr. G. Baretton}

Institut für Pathologie, Universitätsklinikum "Carl Gustav Carus", TU Dresden Fetscherstr. 74, 01307 Dresden Gustavo.Baretton@uniklinikum-dresden.de

\section{Allgemeines}

Angabe eines Interessenkonflikts im Manuskript (weitere Informationen auf der Seite „Erklärung zum Interessenkonflikt")

$\square$ Manuskript immer als Datei schicken (.doc oder .rtf, keine PDF-Dateien)

Gesamtumfang: 12 Manuskriptseiten (Times New Roman, 12 pt, 2-zeilig) inkl. Literatur, Tabellen und Abbildungslegenden (entspricht ca. 25000 Zeichen, inkl. Leerzeichen)

Für die Angaben von Maßeinheiten bitte das SI-System verwenden

$\square$ Abkürzungen im Text erläutern, ggf. Abkürzungsverzeichnis erstellen

\section{Manuskriptaufbau}

Komplette Anschrift des Korrespondenzautors mit Tel.-Nr., Fax, E-Mail sowie Portraitfoto

Kurzer, prägnanter Beitragstitel (ca. 50 Zeichen), ggf. erläuternder Untertitel

Deutsche Zusammenfassung (max. 1200 Zeichen, inkl. Leerzeichen). Bei Überschreitung behält sich der Verlag Kürzungen vor

5 deutsche Schlüsselwörter

Englischer Titel

Englisches Abstract (max. 1200 Zeichen, inkl. Leerzeichen). Abstract und Zusammenfassung sollen inhaltlich identisch sein; möglichst einheitlich British English oder American English verwenden

5 englische Keywords zur besseren Auffindbarkeit in Datenbanken unter Verwendung der "Medical Subject Heading (MeSH)"-Liste des Index Medicus (http://www.nlm. nih.gov/mesh/authors.html)

Kurze Hinführung zum Thema (max.600 Zeichen)

Prägnante und möglichst kurze Zwischenüberschriften (max. 50 Zeichen). 4 Hierachien sind möglich

Fazit für die Praxis (max. 1000 Zeichen, inkl. Leerzeichen)

\section{Literaturverzeichnis}

Maximal 30 Literaturstellen in alphabetischer Reihenfolge und durchnummeriert

$\square$ Zitatnummern im Text in eckige Klammern setzen

Bei Benutzung von „Endnote“: bitte Literaturstyling "Springer Fachzeitschrift MedizinPsychologie" verwenden

Zeitschriftentitel nach Index Medicus abkürzen

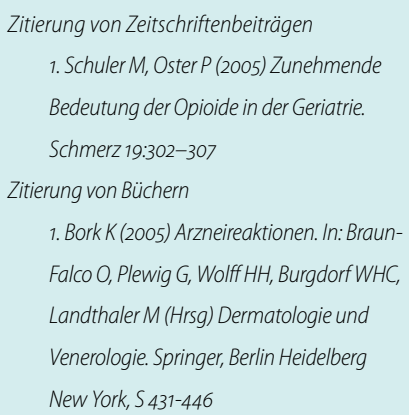

\section{Abbildungen}

Max. 8 Abbildungen mit kurzen Legenden sind möglich

$\square$ Auf alle Abbildungen im Text verweisen

$\square$ Abbildungen entsprechend der Reihenfolge im Text durchnummerieren

Deutsche Abbildungsbeschriftung

Formate: tif, jpg, ppt, eps

Auflösung mind. $300 \mathrm{dpi}$

Unbefristete Abdruckgenehmigung (print/online/offline) für Abbildungen aus Fremdpublikationen

$\square$ Einverständniserklärung identifizierbarer Personen

\section{Videomaterial}

Formate: $640 * 480$ (4:3) Pixel, Container MOV, Video-Codec MPEG-4, Audio-Codec ACC, 96kbps, $25 \mathrm{fps}$

\section{Tabellen}

Aussagekräftige Tabellen mit kurzen Tabellenüberschriften sind erwünscht

Auf alle Tabellen im Text verweisen

Tabellen entsprechend der Reihenfolge im Text durchnummerieren 